\title{
Collagenous colitis with unique colonoscopic
} findings

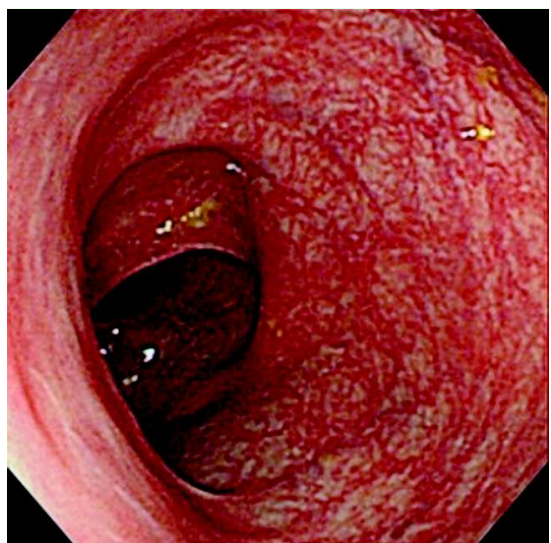

Fig. 1 Colonoscopy showing the crowded vascularity of the colonic mucosa.

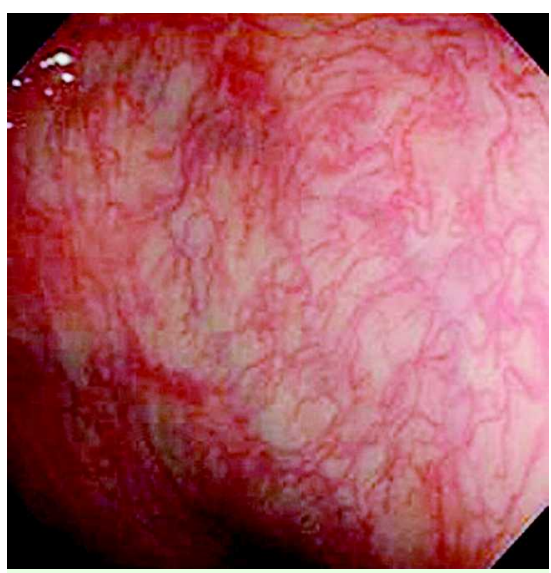

Fig. 2 Close observation of the colonic mucosa showing the dilated, circling or winding blood capillaries.

A 66-year-old Japanese woman with systemic lupus erythematosus (SLE) presented with chronic watery diarrhea, which began 8 months after SLE diagnosis. She was given oral medications such as loperamide but they were unsuccessful. Finally she was admitted for evaluation of the chronic diarrhea, which was then of 4 months' duration. Colonoscopy was performed and showed numerous crowded, small, dilated, circling or winding blood vessels on the mucosal surface of the entire colon, but especially the transverse colon, appearing like a spider web ( Fig. 1,2). A surprising finding was a 20 -cm-long linear ulcer or scarlike area of mucosal damage without bleeding in the descending colon

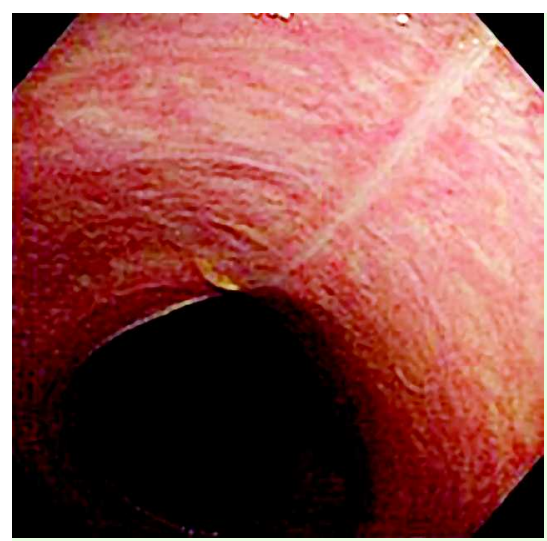

Fig. 3 Mucosa of the descending colon showing the linear ulcer or scar-like lesion.

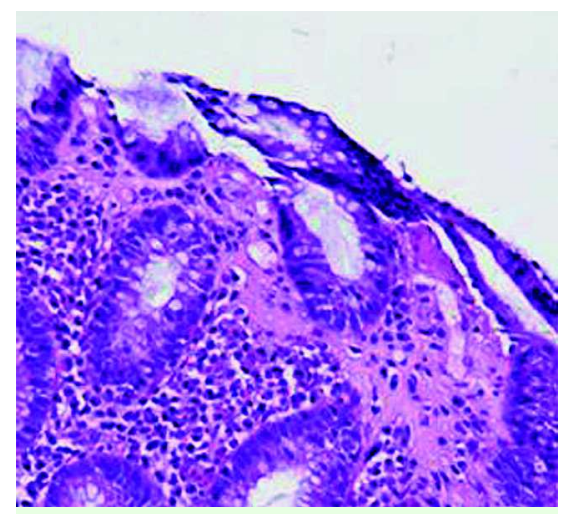

Fig. 4 Biopsy showing subepithelial collagen deposits.

( Fig. 3). Biopsies were taken from the cecum, ascending colon, transverse colon, the ulcer-like lesion of the descending colon, the sigmoid colon, and the rectum. Histopathology results indicated that all the biopsies contained a subepithelial collagen band, compatible with a diagnosis of collagenous colitis ( $\bullet$ Fig. 4). The patient was started on oral mesalamine, which resulted in fewer bowel movements. Finally her diarrhea ceased. Follow-up colonoscopy 2 months later revealed normal colonic mucosal vessels and a clearly diminished linear scar-like lesion.

Collagenous colitis is a disease that generally presents with chronic diarrhea, but without specific radiographic or endoscopic findings. Diagnosis is usually on the basis of histopathological evidence of collagen bands in the subepithelium of the colon [1]. Endoscopic images of collagenous colitis are rarely available today. A few previous collagenous colitis case reports have described abnormal endoscopic findings, with one report of a crowded tortuous vascular pattern in a Japanese woman [2] and another report of colonic linear laceration with bleeding in a Caucasian woman [3]. This case report highlights the possibility of diagnosing collagenous colitis using specific colonoscopic findings, a method that may become even more important given the dramatic development of electronic endoscopy.

Endoscopy_UCTN_Code_CCL_1AD_2AD

\section{Y. Hashimoto, Y. Endo, Y. Kuroki, H. Yoshikumi, M. Yoshiba} Department of Gastroenterology, Fujigaoka Hospital, Showa University, Yokohama, Japan

\section{References}

1 Kingham JG. Microscopic colitis. Gut 1991; 32: 234

2 Sato S, Matsui Tet al. Endoscopic abnormalities in a Japanese patient with collagenous colitis. J Gastroenterol 2003; 38: 812 - 813

3 Cruz-Correa M, Milligan F, Giardiello FM et al. Collagenous colitis with mucosal tear on endoscopic insufflation: a unique presentation. Gut 2002; 51: 600

Bibliography

DOI 10.1055/s-2007-995699

Endoscopy 2008; 40: E162

(c) Georg Thieme Verlag KG Stuttgart · New York . ISSN 0013-726X

\section{Corresponding author}

\section{Y. Hashimoto, MD}

Department of Gastroenterology

Fujigaoka Hospital

Showa University

1-30 Aoba-ku

Fujigaoka

Yokohama

Japan 227-8501

Fax: +81-045-9713824

yu-hashimoto@showa-university-fujigaoka.gr.jp 